\title{
Manipulation of single electron spin in a GaAs quantum dot through the application of geometric phases: The Feynman disentangling technique
}

\author{
Sanjay Prabhakar, ${ }^{1,2}$ James Raynolds, ${ }^{1}$ Akira Inomata, ${ }^{3}$ and Roderick Melnik ${ }^{2,4}$ \\ ${ }^{1}$ College of Nanoscale Science and Engineering, University at Albany, State University of New York \\ ${ }^{2} M^{2} N e T$ Laboratory, Wilfrid Laurier University, Waterloo, ON, N2L 3C5 Canada \\ ${ }^{3}$ Department of Physics, University at Albany, State University of New York \\ ${ }^{4}$ BCAM, Bizkaia Technology Park, 48160 Derio, Spain
}

(Dated: September 12, 2018)

\begin{abstract}
The spin of a single electron in an electrically defined quantum dot in a 2DEG can be manipulated by moving the quantum dot adiabatically in a closed loop in the $2 \mathrm{D}$ plane under the influence of applied gate potentials. In this paper we present analytical expressions and numerical simulations for the spin-flip probabilities during the adiabatic evolution in the presence of the Rashba and Dresselhaus linear spin-orbit interactions. We use the Feynman disentanglement technique to determine the non-Abelian Berry phase and we find exact analytical expressions for three special cases: (i) the pure Rashba spin-orbit coupling, (ii) the pure Dresselhause linear spin-orbit coupling, and (iii) the mixture of the Rashba and Dresselhaus spin-orbit couplings with equal strength. For a mixture of the Rashba and Dresselhaus spin-orbit couplings with unequal strengths, we obtain simulation results by solving numerically the Riccati equation originating from the disentangling procedure. We find that the spin-flip probability in the presence of the mixed spin-orbit couplings is generally larger than those for the pure Rashba case and for the pure Dresselhaus case, and that the complete spin-flip takes place only when the Rashba and Dresselhaus spin-orbit couplings are mixed symmetrically.
\end{abstract}

\section{INTRODUCTION}

Geometric phases abound in physics and their study has attracted considerable attention since the seminal work of Berry 1.2 In recent years a number of researchers have shown their interest in the geometric phases associated with single- and few-spin systems for potential applications in the field of quantum computing and noncharge based logic $\underline{3}^{-\underline{5}}$ One interesting proposal is the notion that the spin of a single electron trapped in an electrostatically defined 2D quantum dot can be manipulated through the application of gate potentials by moving the center of mass of a quantum dot adiabatically in a closed loop and inducing a non-Abelian matrix Berry phase $\frac{6}{6} \mathrm{~A}$ recent work shows that the Berry phases can be changed dramatically by the applications of gate potentials and may be detected in an interference experiment $\underline{\underline{z}}$

In the present paper, we study the non-Abelian unitary operator of the spin states during the adiabatic motion of a single electron spin. The non-Abelian nature here stems from the spin-orbit coupling of an electron in two dimensions. The evolution operator which gives rise to the Berry phase is not easy to evaluate as it contains non-commuting operators. In 1951, Feynman ${ }^{8}$ developed an operator calculus for quantum electrodynamics, in which he devised a way to disentangle the evolution operator involving non-commuting operators. In 1958, Popov ${ }^{9}$ applied the operator calculus, combined with group-theoretical considerations, to the spin rotation for a particle with a magnetic moment in an external magnetic field to obtain exact transition probabilities between the initial and final spin states. In a way similar to Popov's we employ the Feynman technique to disentangle the evolution operator for a quantum dot with the Rashba and Dresselhaus spin-orbit couplings and derive analytical expressions for spin transition probabilities. In particular, we obtain exact closed form expressions for three specific cases: (i) the pure Rashba spinorbit coupling $\frac{10}{\underline{1}}$ (ii) the pure linear Dresselhaus spinorbit coupling, 11 and (iii) the symmetric combination of the Rashba and Dresselhaus spin-orbit couplings. This approach provides us a convenient numerical scheme for an arbitrary mixing of the two types of spin-orbit couplings via a Riccati equation. $\frac{12}{2}$ An interesting result we find is that the spin-flip probability for the case of an arbitrary mixture of the Rashba and the Dresselhaus spinorbit couplings is generally greater than that for the case where either the Rashba or the Dresselhaus interaction acts alone. Furthermore, we see that the complete spin precession occurs only when the Rashba spin-orbit coupling and the Dresselhaus spin-orbit coupling are equal in strength.

The work of Berry teaches that if parameters contained in the Hamiltonian of a quantal system are adiabatically carried around a closed loop an extra geometric phase (Berry phase) is induced in addition to the familiar dynamical phase $\frac{1,2}{12}$ A slow variation of such parameters along a closed path $C$ will return the system to its original energy eigenstate with an additional phase factor $\exp \left\{i \gamma_{n}(C)\right\}$. More specifically, the state acquires phases after a period of the cycle $T$ as

$$
\left|\Psi_{n}(T)\right\rangle=\exp \left\{-\frac{i}{\hbar} \int_{0}^{T} E_{n}(t) d t\right\} \cdot \exp \left\{i \gamma_{n}(C)\right\}\left|\psi_{n}\right\rangle .
$$

However this equation applies only to non-degenerate states. The detailed numerical and analytical calculations of Berry phase $\gamma_{n}(C)$ for the Hamiltonian of a quan- 
tum dot in 2D plane for different non-degenerate eigen states are explained in Ref. 13. The system of interest here (a single spin in a 2D electrically defined quantum dot) is degenerate ${ }^{14,15}$ for which (1) is not directly applicable. In the formulation developed by Wilczek and others $^{2,16}$ for degenerate cases, the geometric phase factor is replaced by a non-Abelian unitary operator $U_{a b}$ acting on the initial states within the subspace of degeneracy. The evolution equation of the state is modified in the form,

$$
\left|\Psi_{n, a}(t)\right\rangle=\exp \left\{-\frac{i}{\hbar} \int_{0}^{t} E(t) d t\right\} \sum_{b} U_{a b}(t)\left|\psi_{n, b}\right\rangle
$$

where $a$ and $b$ are the labels for degeneracy. The nonAbelian unitary operator can be expressed in the form,

$$
U_{a b}(t)=T \exp \left\{-\frac{i}{\hbar} \int_{0}^{t} \mathbf{A}_{a b}\left(t^{\prime}\right) \cdot \dot{\mathbf{R}} d t^{\prime}\right\}
$$

where $T$ signifies the time-ordering, and

$$
\mathbf{A}_{a b}=-i \hbar\left\langle\psi_{n, a}\left|\nabla_{\mathbf{R}}\right| \psi_{n, b}\right\rangle
$$

$\mathbf{R}$ and $\nabla_{\mathbf{R}}$ being a vector and the gradient in parameter space, respectively. In general, the geometric phase transformation $U_{a b}(t)$ of (3) in parameter space contains non-commuting operators and time-dependent parameters. It is possible to view the parameter-dependent evolution in the subspace of degeneracy as a non-Abelian local gauge transformation. Correspondingly $\mathbf{A}_{a b}$ in (4) may be seen as a non-Abelian gauge connection (or the Yang-Mills fields).

Although it is not straightforward to construct the non-Abelian gauge connection, we consider the following observation instructive for the case where the parameter space coincides with the configuration space. Suppose the Hamiltonian of a system is given by

$$
H=\frac{1}{2 m}(\mathbf{P}-\mathbf{A})^{2}+V(\mathbf{r})
$$

The energy eigenequation $H\left|\psi_{n}\right\rangle=E_{n}\left|\psi_{n}\right\rangle$ remains invariant under the local (position-dependent) gauge transformation,

$$
\left|\psi_{n}\right\rangle \rightarrow\left|\psi_{n}^{\prime}\right\rangle=\bar{U}\left|\psi_{n}\right\rangle, \quad \mathbf{A} \rightarrow \mathbf{A}^{\prime}=\bar{U} \mathbf{A} \bar{U}^{\dagger}+i \hbar \bar{U} \nabla \bar{U}^{\dagger}
$$

If we choose such a gauge that the transformed vector potential vanishes, that is, $\mathbf{A}^{\prime}=0$, then the transformation operator is to be of the form,

$$
\bar{U}=\exp \left\{-\frac{i}{\hbar} \oint_{c} \mathbf{A} \cdot d \mathbf{r}\right\}
$$

In other words, this transformation will "gauge away" the vector potential from the Hamiltonian (5). Conversely, if the state with the vanishing gauge is taken to be the initial state, the final state with an arbitrary gauge $\mathbf{A}$ is obtained by the inverse gauge transformation,
$\left|\psi_{n}\right\rangle=\bar{U}^{-1}\left|\psi_{n}^{\prime}\right\rangle$. Moreover, if the inverse gauge process is time-dependent via the variation of position, then the evolution operator is given by

$$
U(t)=\bar{U}^{-1}(t)=T \exp \left\{\frac{i}{\hbar} \int_{0}^{t} \mathbf{A} \cdot \dot{\mathbf{r}} d t\right\} .
$$

This observation will be useful for our discussion on the Berry phase associated with the spin-orbit coupling.

A matrix element of the evolution operator gives the transition amplitude (propagator) from an initial state to the final state, which is usually evaluated by approximation. For instance, the propagator for the spin-orbit interaction has been calculated semiclassically in a different context by Feynman's path integral represented in coherent states. 17

In Sec. 2, we treat the phase transformation (3) as a gauge transformation, and employ Feynman's disentangling technique, rather than Feynman's path integral, to evaluate the time-ordered exponential for the spin-orbit coupling Hamiltonian. Use of Feynman's disentangling method in Popov's version ${ }^{18}$ enables us to obtain analytical and numerical results for the spin transition probabilities without approximation. In Sec. 3, we plot the spin-flip probability versus the rotation angle, and compare the data for the pure Rashba, the pure Dresselhaus, and mixed cases. Sec. 4 is devoted in deriving analytical expressions of the non-Abelian Berry phase (the adiabatic evolution operator as a $2 \times 2$ matrix) for the pure Rashba and the pure Dresselhaus coupling.

\section{SPIN TRANSITION PROBABILITIES VIA FEYNMAN DISENTANGLING METHOD}

To discuss the revolution of spin that induces a geometric phase, we consider a GaAs quantum dot formed in the plane of a two-dimensional electron gas (2DEG), the center of mass of which moves adiabatically along a closed path under the influence of applied potentials 6 The single-electron Hamiltonian in 2DEG (in the $x y$ plane) may be written in the form,

$$
H=\frac{1}{2 m} \mathbf{P}^{2}+H_{S O},
$$

where $m$ is the effective mass. The first term is the kinetic energy in two dimensions. Evidently, $\mathbf{P}^{2}=P_{x}^{2}+P_{y}^{2}$. The second term is the spin-orbit ( $\mathrm{SO}$ ) coupling Hamiltonian in linear approximation,

$$
H_{S O}=2 \alpha\left(P_{y} S_{x}-P_{x} S_{y}\right)-2 \beta\left(P_{x} S_{x}-P_{y} S_{y}\right) .
$$

Here $\mathbf{S}$ is the spin operator whose components obey the $S U(2)$ algebra (see, e.g., Ref. 19):

$$
\left[S_{+}, S_{-}\right]=2 S_{0}, \quad\left[S_{0}, S_{ \pm}\right]= \pm S_{ \pm}
$$

where $S_{ \pm}=S_{x} \pm i S_{y}$ and $S_{0}=S_{z}$. The spin-orbit Hamiltonian (10) consists of the Rashba coupling whose 


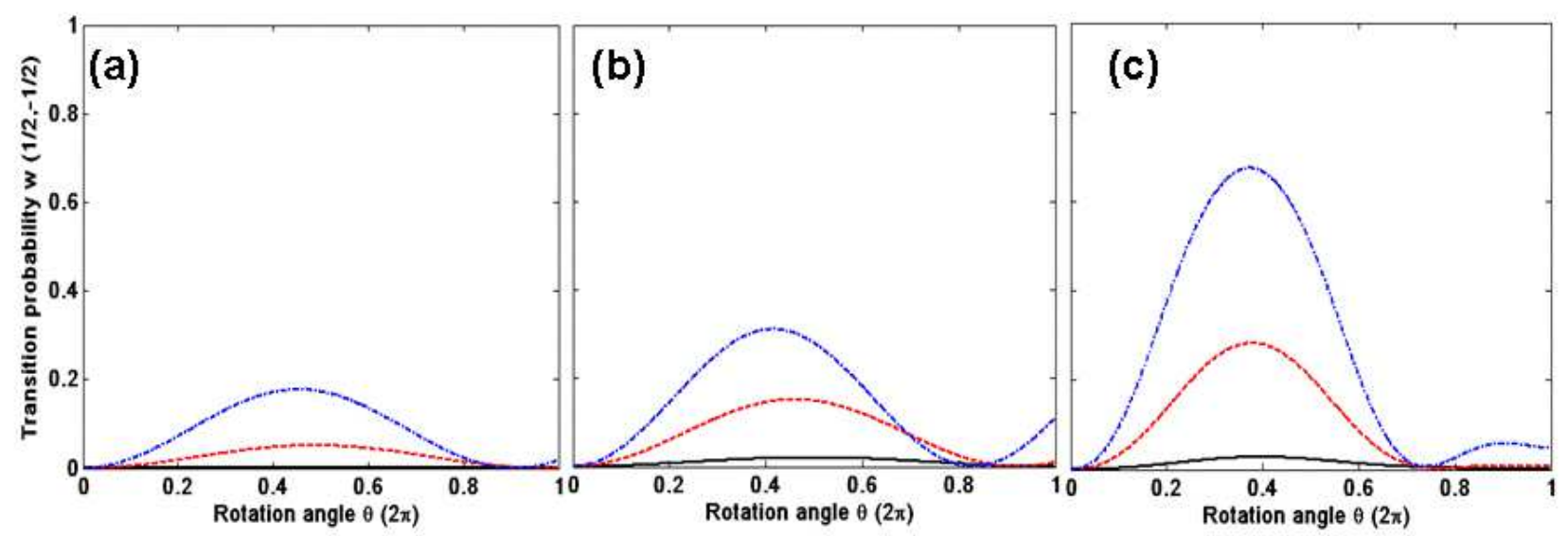

FIG. 1. (color online) Transition probability, $w_{1 / 2,-1 / 2}$ vs. $\theta$ for three cases: (a) pure Rashba $(\beta=0)$, (b) pure Dresselhaus $(\alpha=0)$, and (c) mixed (non-zero $\alpha$ and $\beta$ ) spin-orbit interactions. The orbital radius is $60 \mathrm{~nm}$. The three curves represent the following electric field strengths: $1 \times 10^{5} \mathrm{~V} / \mathrm{cm}$ (solid black line), $5 \times 10^{5} \mathrm{~V} / \mathrm{cm}$ (dashed red line), and $1 \times 10^{6} \mathrm{~V} / \mathrm{cm}$ (dotted-dashed blue line) respectively.

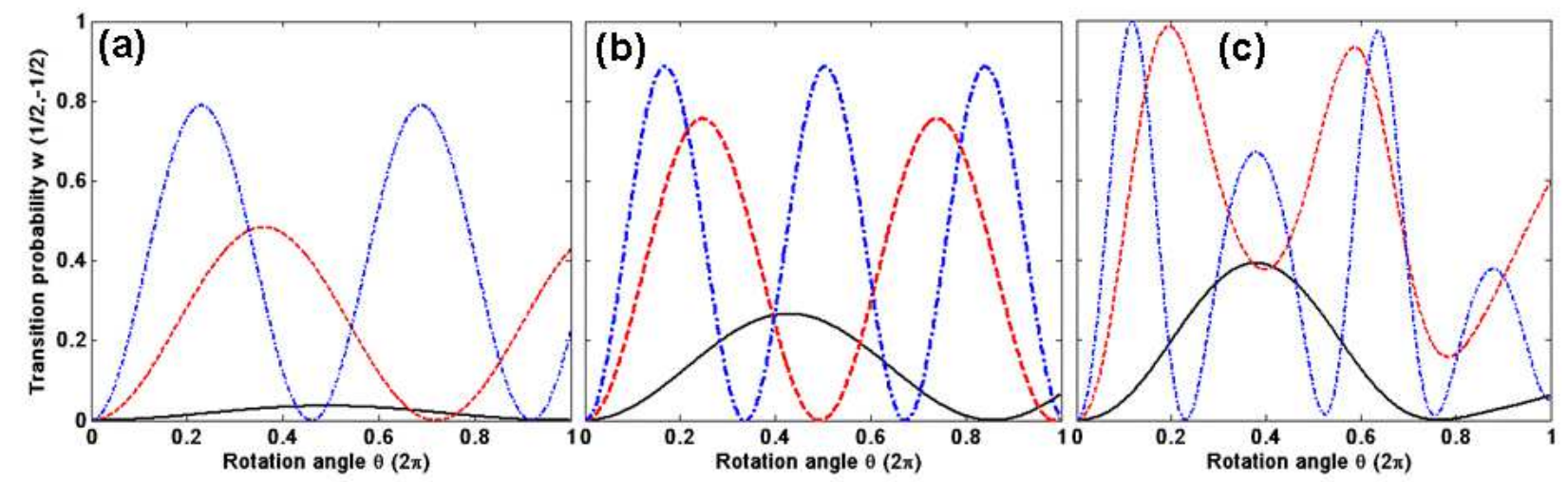

FIG. 2. (color online) Transition probability $w_{1 / 2,-1 / 2}$ vs. $\theta$ for the following cases: (a) pure Rashba $(\beta=0)$, (b) pure Dresselhaus $(\alpha=0)$, and (c) mixed (non-zero $\alpha$ and $\beta$ ). The orbit radius is chosen to be $250 \mathrm{~nm}$ and the following values of the electric field are considered: $1 \times 10^{5} \mathrm{~V} / \mathrm{cm}$ (solid black line), $5 \times 10^{5} \mathrm{~V} / \mathrm{cm}$ (dashed red line), and $1 \times 10^{6} \mathrm{~V} / \mathrm{cm}($ dotted-dashed blue line)

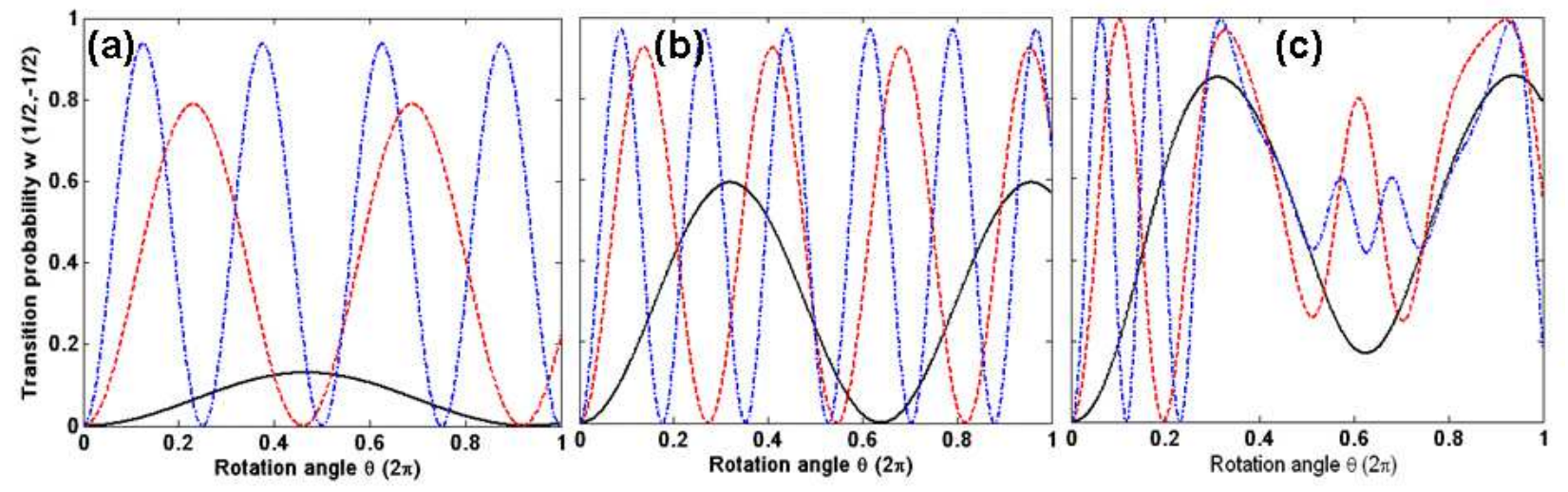

FIG. 3. (color online) Transition probability $w_{1 / 2,-1 / 2}$ vs. $\theta$ for the following cases: (a) pure Rashba $(\beta=0)$, (b) pure Dresselhaus $(\alpha=0$ ), and (c) mixed (non-zero $\alpha$ and $\beta$ ). The orbit radius was chosen to be $500 \mathrm{~nm}$ and the following values of the electric field were chosen: $1 \times 10^{5} \mathrm{~V} / \mathrm{cm}$ (solid black line), $5 \times 10^{5} \mathrm{~V} / \mathrm{cm}$ (dashed red line), and $1 \times 10^{6} \mathrm{~V} / \mathrm{cm}($ dotted-dashed blue line). 


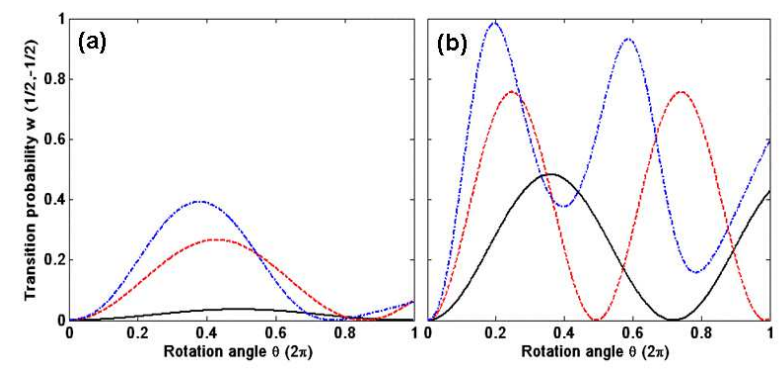

FIG. 4. (color online) Transition probability $w_{1 / 2,-1 / 2}$ vs. $\theta$ for the following cases: pure Rashba $(\beta=0$ : dotted-dashed blue line), pure Dresselhaus ( $\alpha=0$ : dashed red line) and mixed (non-zero $\alpha$ and $\beta$ : solid black line). The orbit radius was chosen to be $250 \mathrm{~nm}$ and the following values of the electric field were chosen: (a) $E=1 \times 10^{5} \mathrm{~V} / \mathrm{cm}$ and, (b) $E=5 \times 10^{5} \mathrm{~V} / \mathrm{cm}$.

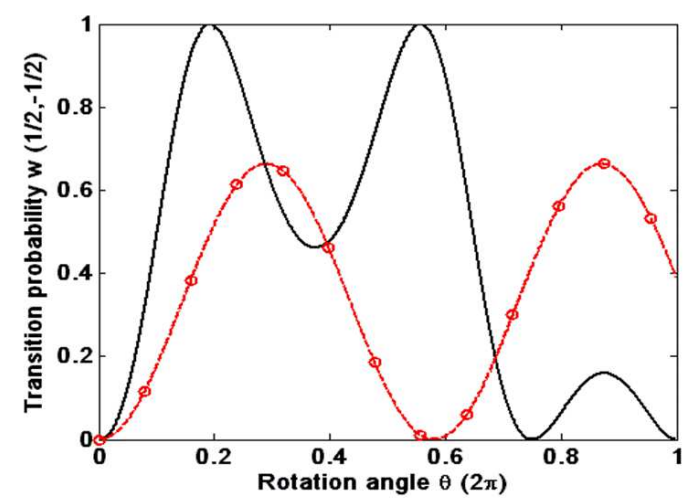

FIG. 5. (color online) Transition probability $w_{1 / 2,-1 / 2}$ vs. $\theta$ for $\alpha=\beta$. Physically, this situation occurs for electric field strength given by $E=3.02 \times 10^{6} \mathrm{~V} / \mathrm{cm}$. Here the solid black line represents for both Rashba and Dresselhaus spin-orbit coupling effects whereas the dashed red line represents only for Dresselhaus spin-orbit coupling effect and open red circles represents only for Rashba spin-orbit coupling effect. Here we choose $60 \mathrm{~nm}$ orbit radius.

strength is characterized by parameter $\alpha$ and the linear Dresselhaus coupling with $\beta$. These coupling parameters are dependent on the electric field $E$ of the quantum well confining potential (i.e., $E=-\partial V / \partial z$ ) along z-direction at the interface in a heterojunction as

$$
\alpha=\frac{e}{\hbar} a_{R} E, \quad \beta=\frac{0.7794 \gamma_{c}}{\hbar}\left(\frac{2 m e}{\hbar^{2}}\right)^{2 / 3} E^{2 / 3},
$$

where $a_{R}=4.4 \AA^{2}$ and $\gamma_{c}=26 \mathrm{eV} \AA^{3}$ for the GaAs quantum dot $\stackrel{14}{1}$ The quantum well confining potential (i.e., $E=-\partial V / \partial z)$ along z-direction is not symmetric in III-V type semiconductor $\frac{15}{1 t}$ It means, the formation of quantum dot at the interface of III-V type semiconductor in the plane of $2 \mathrm{DEG}$ is asymmetric.

Now we look for the evolution operator (8) for the case of spin-orbit coupling. It has been known that the linear spin-orbit term in (9) can be gauged away $\underline{20,21}$ In fact,

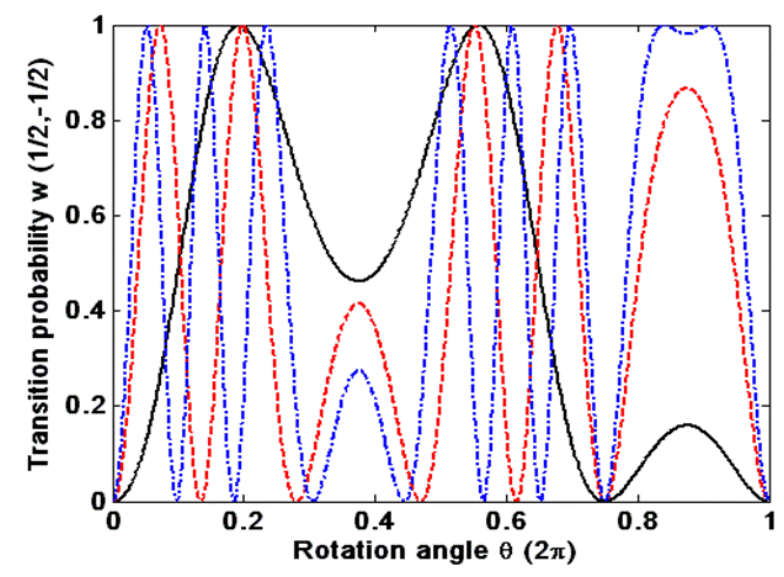

FIG. 6. (color online) Transition probability $w_{1 / 2,-1 / 2}$ vs. $\theta$ for $\alpha=\beta$. Physically, this situation occurs for electric field strength given by $E=3.02 \times 10^{6} \mathrm{~V} / \mathrm{cm}$. The following orbit radii were chosen: $60 \mathrm{~nm}$ (solid black line), $175 \mathrm{~nm}$ (dashed red line), and, $250 \mathrm{~nm}$ (dotted-dashed blue line).

the Hamiltonian (9) may be expressed as

$$
H=\frac{1}{2 m}(\mathbf{P}-\mathbf{A})^{2}-V_{0},
$$

where

$$
\mathbf{A}=2 m\left(\begin{array}{c}
\alpha S_{y}+\beta S_{x} \\
-\alpha S_{x}-\beta S_{y}
\end{array}\right)
$$

and

$$
V_{0}=m \hbar^{2}\left(\alpha^{2}+\beta^{2}\right) .
$$

If the semiclassical momentum $\mathbf{P}=m \dot{\mathbf{r}}$ is used for the adiabatic evolution, then the spin-orbit gauge connection is related to the SO Hamiltonian (10),

$$
\mathbf{A} \cdot \dot{\mathbf{r}}=-H_{S O}
$$

Assuming that the spin-orbit coupling is adiabatically introduced into the initial state, we obtain via (8) the evolution operator of the form,

$$
U(t)=T \exp \left\{-\frac{i}{\hbar} \int_{0}^{t} H_{S O}\left(t^{\prime}\right) d t^{\prime}\right\}
$$

which we shall evaluate by utilizing the Feynman disentangling method. This form of the evolution operator is commonly employed for Berry's phase associated with the spin-orbit interaction. $\underline{\underline{6} .15}$

Before disentangling, we note that the SO Hamiltonian (10) may also be expressed as

$$
H_{S O}=H_{+} S_{+}+H_{-} S_{-}
$$

with

$$
H_{ \pm}=\left(\alpha P_{y}-\beta P_{x}\right) \mp i\left(\beta P_{y}-\alpha P_{x}\right)
$$


Suppose the quantum dot orbits around a closed circular path of radius $R_{0}$ in the $x-y$ plane under the influence of gate potentials, so that $\mathbf{r}=R_{0}(\cos \omega t, \sin \omega t, 0)$. Then the semiclassical momentum $\mathbf{P}=m \dot{\mathbf{r}}$ has components,

$$
P_{x}=-R_{0} m \omega \sin \omega t, P_{y}=R_{0} m \omega \cos \omega t, P_{z}=0 .
$$

Substitution of (20) into (19) yields

$$
H_{ \pm}=R_{0} m \omega\left(\alpha e^{\mp i \omega t} \mp i \beta e^{ \pm \omega t}\right) .
$$

Since $S_{+}$and $S_{-}$do not commute, the evaluation of the time-ordered exponential for the evolution operator (17) is cumbersome.

We now turn to a discussion of the Feynman disentangling technique and its application to the present problem. For the case where the Hamiltonian is given by

$$
H=\alpha(t) A+\beta(t) B+\gamma(t) C+\cdots,
$$

where $A, B, C, \ldots$ are noncommuting operators, and $\alpha, \beta, \gamma, \ldots$ are time-dependent parameters, Feynman $\underline{8}$ devised an operator calculus by which the time-ordered exponential can be disentangled in the form

$$
U(t)=e^{a(t) A} e^{b(t) B} e^{c(t) C} \cdots,
$$

where $a(t), b(t), c(t), \ldots$ are time-dependent coefficients which can be determined by solving relevant differential equations. This procedure is referred to as the Feynman disentangling method. $\underline{18}^{18}$

Here we apply Feynman's method for disentangling the time-ordered exponential in (17) with the Hamiltonian (10). First we rewrite the Hamiltonian (10) as

$$
H_{S O}=\xi S_{+}+\left(H_{+}-\xi\right) S_{+}+H_{-} S_{-},
$$

where $\xi$ is a time-dependent function to be determined appropriately. According to Feynman's procedure, the evolution operator may be put into the form,

$U(t)=e^{a(t) S_{+}} \exp \left\{\frac{1}{i \hbar} \int_{0}^{t} d t^{\prime}\left[\left(H_{+}-\xi\right) S_{+}^{\prime}+H_{-} S_{-}^{\prime}\right]\right\}$,

where

$$
\begin{gathered}
a(t)=\frac{1}{i \hbar} \int_{0}^{t} \xi\left(t^{\prime}\right) d t^{\prime}, \\
S_{+}^{\prime}=e^{-a S_{+}} S_{+} e^{a S_{+}}=S_{+}
\end{gathered}
$$

and

$$
S_{-}^{\prime}=e^{-a S_{+}} S_{+} e^{a S_{+}}=S_{-}-2 a S_{0}-a^{2} S_{+} .
$$

Substituting (27) and (28) into (25) and choosing $\xi(t)$ such that the coefficient of $S_{+}$in the integrand vanishes, we get

$U(t)=e^{a(t) S_{+}} T \exp \left\{\frac{1}{i \hbar} \int_{0}^{t} d t^{\prime}\left[-2 a H_{-} S_{0}+H_{-} S_{-}\right]\right\}$, in which the term containing $S_{+}$is disentangled. In a similar fashion, we disentangle the time-ordered exponential involving the mutually non-commuting operators $S_{0}$ and $S_{-}$by letting

$$
\begin{aligned}
U(t)= & e^{a(t) S_{+}} e^{b(t) S_{0}} \\
& T \exp \left\{\frac{1}{i \hbar} \int_{0}^{t} d t^{\prime}\left[\left(-2 a H_{-}-\eta\right) S_{0}^{\prime \prime}+H_{-} S_{-}^{\prime \prime}\right]\right\},
\end{aligned}
$$

where

$$
\begin{gathered}
b(t)=\frac{1}{i \hbar} \int_{0}^{t} \eta\left(t^{\prime}\right) d t^{\prime}, \\
S_{0}^{\prime \prime}=e^{-b S_{0}} S_{0} e^{b S_{0}}=S_{0}
\end{gathered}
$$

and

$$
S_{-}^{\prime \prime}=e^{-b S_{0}} S_{-} e^{b S_{0}}=S_{-} e^{b} .
$$

Again choosing $\eta(t)=-2 a H_{-}$, we reduce the evolution operator (25) into the completely disentangled form,

$$
U(t)=e^{a(t) S_{+}} e^{b(t) S_{0}} e^{c(t) S_{-}},
$$

where

$$
\begin{gathered}
a(t)=\frac{1}{i \hbar} \int_{0}^{t}\left[H_{+}\left(t^{\prime}\right)-a^{2}\left(t^{\prime}\right) H_{-}\left(t^{\prime}\right)\right] d t^{\prime}, \\
b(t)=-\frac{2}{i \hbar} \int_{0}^{t} a\left(t^{\prime}\right) H_{-}\left(t^{\prime}\right) d t^{\prime}
\end{gathered}
$$

and

$$
c(t)=\frac{1}{i \hbar} \int_{0}^{t} H_{-}\left(t^{\prime}\right) e^{b\left(t^{\prime}\right)} d t^{\prime} .
$$

Although the time-ordered exponential is disentangled, the evaluation of the evolution operator remains incomplete until the coefficients $a(t), b(t)$ and $c(t)$ are determined. In general, the integral equations (35)-(37) or the equivalent differential equations are difficult to solve. In Sec. 4, we shall determine the coefficients and the evolution operator for the pure Rashba, and the pure Dresselhaus coupling.

As it is seen in Appendix A, the spin transition probability depends only on $a(t)$. Therefore the full form of the evolution operator is not needed. To determine $a(t)$, we convert the integral equation (35) together with (19) into a Riccati equation of the form,

$$
\frac{d a}{d t}=-R \omega\left[f(t)+f^{*}(t) a^{2}(t)\right],
$$

where $R=m R_{0} / \hbar$,

$$
f(t)=\beta^{i \omega t}+i \alpha e^{-i \omega t},
$$


and

$$
f^{*}(t)=\beta^{-i \omega t}-i \alpha e^{i \omega t} .
$$

Solving (38) for $a(t)$, we can obtain the spin transition probabilities, $w_{s, s^{\prime}}$. In particular, the transition probabilities from spin $1 / 2$ to $\pm 1 / 2$ are calculated by

$$
w_{1 / 2,1 / 2}=\frac{1}{1+|a|^{2}}, \quad w_{1 / 2,-1 / 2}=\frac{|a|^{2}}{1+|a|^{2}} .
$$

\section{NUMERICAL ANALYSIS}

As it is shown in Appendix B, exact solutions of the Riccati equation (38) can be obtained only for special cases, which include those for (i) the Rashba limit $(\beta=0)$, (ii) the Dresselhaus limit $(\alpha=0)$ and (iii) the symmetric mixture of the two couplings $(\alpha=\beta)$. The spin-flip probabilities obtained in Appendix B for exactly solvable cases (with $\theta=\omega t$ ) are:

(i) The Rashba limit $(\alpha \neq 0, \beta=0)$ :

$$
w_{1 / 2,-1 / 2}^{R}=\frac{4 R^{2} \alpha^{2}}{1+4 R^{2} \alpha^{2}} \sin ^{2}\left(\frac{1}{2} \sqrt{1+4 R^{2} \alpha^{2}} \theta\right) ;
$$

(ii) The Dresselhaus limit $(\alpha=0, \beta \neq 0)$ :

$$
w_{1 / 2,-1 / 2}^{D}=\frac{4 R^{2} \beta^{2}}{1+4 R^{2} \beta^{2}} \sin ^{2}\left\{\frac{1}{2} \sqrt{1+4 R^{2} \beta^{2}} \theta\right\} ;
$$

(iii) The symmetric Rashba-Dresselhaus limit ( $\alpha=$ $\beta \neq 0)$ :

$$
w_{1 / 2,-1 / 2}^{\text {sym }}=\sin ^{2}\{\sqrt{2} \alpha R(\sin \theta-\cos \theta+1)\} .
$$

For an arbitrarily mixed Rashba-Dresselhaus coupling (mixed R-D), the Riccati equation (38) is not exactly solvable. Therefore numerical analysis is needed. In the below we treat the mixed R-D coupling $(\alpha \neq \beta)$ and the symmetric $\mathrm{R}=\mathrm{D}$ coupling $(\alpha=\beta)$ separately.

Comparison of the Rashba coupling, the Dresselhaus coupling and the mixed R-D coupling: Figs. 11, 2 and 3 plot the spin-flip probability $w_{1 / 2,-1 / 2}$ versus the rotation angle $\theta=\omega t$ in the unit of $2 \pi$ for the orbit radius $R_{0}=60 \mathrm{~nm}, 250 \mathrm{~nm}$, and $500 \mathrm{~nm}$, respectively. The plots of (a), (b) and (c) in these figures correspond to (a) the pure Rashba case $(\beta=0)$, (b) the pure Dresselhaus case $(\alpha=0)$ and (c) the mixed R-D case $(\alpha \neq 0, \beta \neq 0)$, respectively. The three different values of the electric field $E=1 \times 10^{5} \mathrm{~V} / \mathrm{cm}, 5 \times 10^{5}$ $\mathrm{V} / \mathrm{cm}$, and $1 \times 10^{6} \mathrm{~V} / \mathrm{cm}$, are chosen for the curves in each figure, solid black, dashed red, and dotted-dashed blue, respectively. The symmetric case $(R=D)$ will be examined separately with Figs. 5 and 6 .

The curves for (a) the pure Rashba case and (b) the pure Dresselhaus case are obtained from the exact results
(42) and (43). As it is obvious from these equations, the spin-flip probability increases as the electric field increases via the coupling parameter but remains to be less than unity. Another observation we can make from these plots is that the periods of spin-flip for the pure Rashba coupling and the pure Dresselhaus coupling are different. This is also expected from the analytical results (42) and (43).

The curves in Figs.11(c), 2(c) and 3(c) show the spinflip probability for (c) the mixed R-D case where both $\alpha$ and $\beta$ are not zero and not equal. Note that they are not the results from the exact formula (44) for the symmetric R-D coupling. Since the Riccati equation (38) for arbitrary non-zero $\alpha$ and $\beta$ is not solvable, we carry out numerical simulations by using numerical solutions of (38) in (41). The spin-flip probability for the mixed case is generally larger than the pure cases. Furthermore, it does not reach unity if $\alpha \neq \beta$. In other words, the complete spin-flip is not likely to occur during the entire period of the adiabatic motion along the closed orbit. In the vicinity of the symmetry point $(\alpha=\beta)$, the transition probability becomes very close to unity at certain angles.

Fig. [4 gives a further comparison study of the transition probability for the pure Rashba, the pure Dresselhaus, and the mixed case. In Fig. 4(a), when the electric field is weak, the curve for the mixed case appears to be a superposition of those for the two pure cases. As the electric field increases, the superposition effect becomes obscure as is seen in Fig. 4(b). As the Riccati equation is nonlinear in nature, there is no reason to expect that the mixed case is a superposition of the two pure cases. It is interesting to observe that the mixed case has a better chance to achieve the spin-flip than the pure cases during the period of evolution.

Analysis of the symmetric R-D coupling:- The symmetric mixture of the Rashba and Dresselhaus couplings has been discussed in connection with the persistent spin helix ${ }^{22,23}$ Bernevig et al. ${ }^{22}$ found an exact $S U(2)$ symmetry in the symmetric mixture and predicted the persistent spin helix which is a helical spin density wave with conserved amplitude and phase. Recently spin life time enhancement of two orders of magnitude near the symmetry point $(\alpha=\beta)$ has been reported experimentally, $\underline{24}$

The coupling parameters $\alpha$ and $\beta$ of the Rashba and Dresselhaus interactions are given by (12) for the GaAs quantum dot. The two parameters become equal at $E=3.02 \times 10^{6} \mathrm{~V} / \mathrm{cm}$. For the situation in which the two couplings have equal strength (i.e., $\alpha=\beta$ ), the Riccati equation (38) is exactly solved and the corresponding transition probability is given by (44). In Fig. 5, the spinflip probability versus the angle of rotation along the orbit of radius $60 \mathrm{~nm}$ is plotted at $E=3.02 \times 10^{6} \mathrm{~V} / \mathrm{cm}$ for the pure Rashba case (open red circles), the pure Dresselhaus case (dashed red line), and the symmetric case (solid black line). We see that the symmetric RashbaDresselhaus spin-orbit coupling definitely achieves a spinflip during the adiabatic process whereas the two pure 
cases have less chances. Fig. 6 plots the transition probability of the symmetric R-D case for three different radii of the orbit of the quantum dot: $60 \mathrm{~nm}$ (solid black line), $175 \mathrm{~nm}$ (dashed red line) and $250 \mathrm{~nm}$ (dotted-dashed blue line). It shows that the chance of being in the spin-flip state is enhanced by increasing the orbit radius.

It is important to notice that the complete spin flip takes place only in the symmetric R-D coupling. This may be an indication of the persistent spin helix. Although the assumed orbit of motion is circular, we can regard the motion for a small angle of rotation as linear. Let $\theta=\varepsilon \approx 0$ or $\theta=3 \pi / 2-\varepsilon$. If $\varepsilon$ is small, then $\sin \theta-\cos \theta+1 \approx \varepsilon$, and the exact formula (44) may be approximated by

$$
w_{1 / 2,-1 / 2}^{\text {sym }}=\sin ^{2}\{\sqrt{2} \alpha R \varepsilon\} .
$$

As $\varepsilon$ varies from 0 to $\pi /(2 \sqrt{2} \alpha R)$, the spin-flip probability moves from zero to unity, that is, the spin completes a full precession. For instance, if $R=60 \mathrm{~nm}$, the range $0 \leq \sqrt{2} \alpha R \varepsilon \leq \pi / 2$ corresponds to the portion of the solid black curve for $0 \leq \theta / 2 \pi<0.2$ in Fig. 5. Let $\varepsilon_{s}=\pi /(2 \sqrt{2} \alpha R)$. Then the $R \varepsilon_{s}$ is the distance the electron progresses while the spin precesses by $2 \pi$. Therefore, we may be able to identify this distance with the spin diffusion length $L_{s}$ as

$$
L_{s}=R \varepsilon_{0} / \pi=\frac{1}{2 \sqrt{2} \alpha} .
$$

\section{ANALYTICAL EXPRESSION FOR THE NON-ABELIAN BERRY PHASE}

Applying the Feynman disentangling method, we have been able to reduce the time-ordered evolution operator (17) to the disentangled form (34) with the timedependent scalar functions $a(t), b(t)$ and $c(t)$ obeying the integral equations (35)-(37). It is sometimes convenient to express the evolution operator as a $2 \times 2$ matrix in the spin representation of $S U(2)$. Evidently the $S U(2)$ algebra (11) is satisfied by

$$
S_{+}=\left(\begin{array}{ll}
0 & 1 \\
0 & 0
\end{array}\right), \quad S_{0}=\frac{1}{2}\left(\begin{array}{cc}
1 & 0 \\
0 & -1
\end{array}\right), \quad S_{-}=\left(\begin{array}{ll}
0 & 0 \\
1 & 0
\end{array}\right) .
$$

Using the properties $S_{ \pm}^{2}=0$ and $S_{0}^{2}=1 / 4$, we can write (34) as

$$
U(t)=\left(\begin{array}{ll}
1 & a \\
0 & 1
\end{array}\right)\left(\begin{array}{cc}
e^{b / 2} & 0 \\
0 & e^{-b / 2}
\end{array}\right)\left(\begin{array}{ll}
1 & 0 \\
c & 1
\end{array}\right),
$$

from which immediately follows that

$$
U(t)=\left(\begin{array}{cc}
e^{b / 2}+a c e^{-b / 2} & a e^{-b / 2} \\
c e^{-b / 2} & e^{-b / 2}
\end{array}\right) .
$$

This is the desired matrix representation for the Berry phase, and is used for calculating the spin-flip probabilities in Appendix A.
The expressions (34) and (49) remain formal until the time-dependent functions $a(t), b(t)$ and $c(t)$ are specified. Eq. (35) for $a(t)$ is equivalent to a Riccati equation without whose solution, (36) and (37) cannot be solved for $b(t)$ and $c(t)$. In Appendix B, we show that the Riccati equation can be solved exactly if the function $h(t)$ defined by

$$
h(t)=\frac{\beta^{2}-\alpha^{2}}{2 R\left(\alpha^{2}+\beta^{2}\right)^{3 / 2}}\left[1+\frac{2 \alpha \beta}{\alpha^{2}+\beta^{2}} \sin (2 \omega t)\right]^{-3 / 2}
$$

becomes time-independent $\left(h(t)=h_{0}\right)$. The last restriction (50) is fulfilled only when one of the following conditions is met: $\alpha=0, \beta=0$ or $\alpha=\beta$. This implies that the function $a(t)$ can be determined only for the pure Dresselhaus coupling, the pure Rashba coupling and the symmetric Rashba-Dresselhaus coupling. The result we find for $a(t)$ is

$$
a(t)=\frac{i f}{|f|} \frac{e^{i \phi(t)}-1}{n_{1} e^{i \phi(t)}-n_{2}}
$$

where

$$
\begin{gathered}
f(t)=\beta e^{i \omega t}+i \alpha e^{-i \omega t}, \\
\phi(t)=R \omega\left(n_{1}-n_{2}\right) \int_{0}^{t}\left|f\left(t^{\prime}\right)\right| d t^{\prime} .
\end{gathered}
$$

Here

$$
n_{1}, n_{2}=h_{0} \pm \sqrt{h_{0}^{2}+1}
$$

For convenience, we choose $n_{1}>n_{2}$. A closed form expression for $\phi(t)$ is given in (B12).

In calculating the spin transition probability, all we need is $a(t)$. However, for completing the evolution operator we have also to determine other functions $b(t)$ and $c(t)$ by solving (36) and (37) for the already determined function $a(t)$. As has been mentioned above, the Riccati equation can be solved exactly for the pure Rashba coupling, the pure Dresselhaus coupling and the symmetric Rashba-Dresselhaus coupling. In the two pure couplings, the phase function $\phi(t)$ can be expressed in the form,

$$
\phi(t)=\varphi t
$$

where $\varphi=\sqrt{1+4 \alpha^{2} R^{2}} \omega$ for the Rashba coupling and $\varphi=\sqrt{1+4 \beta^{2} R^{2}} \omega$ for the Dresselhaus coupling. For the symmetric R-D coupling, it cannot be simplified in the form of (55). Therefore, it is difficult to carry out integration in (36) and (37). This means that we have analytical expressions of the adiabatic evolution operator (49) only for the pure Rashba and the pure Dresselhaus cases. For the symmetric R-D coupling, even though we have no analytical expressions for $b(t)$ and $c(t)$, we can calculate the spin-flip probability since $a(t)$ is found in closed form. 
In what follows we provide the results of integration for the pure Rashba coupling and the pure Dresselhaus coupling.

(i) The pure Rashba coupling $(\alpha \neq 0, \beta=0)$ :

In this case, (B2), (B4), (B5) and (B11) yield,

$$
\text { if } /|f|=-e^{i \omega t}, \quad h_{0}=-\frac{1}{2 \alpha R}
$$

and

$$
\phi(t)=\varphi t, \quad \varphi=\omega \sqrt{1+4 \alpha^{2} R^{2}} .
$$

Upon substitution of these results into (B13) we arrive at

$$
a(t)=-e^{i \omega t} \frac{e^{i \varphi t}-1}{n_{1} e^{\varphi t}-n_{2}},
$$

where

$$
n_{1}, n_{2}=-\frac{1}{2 \alpha R} \pm \frac{1}{2 \alpha R} \sqrt{1+4 \alpha^{2} R^{2}} .
$$

From Eq.(36), using

$$
H_{-}=\alpha R \hbar \omega e^{i \omega t},
$$

together with (56), we obtain

$$
e^{b(t)}=\frac{\left(n_{1}-n_{2}\right)^{2} e^{i(\varphi-\omega) t}}{\left(n_{1} e^{i \varphi t}-n_{2}\right)^{2}}
$$

and

$$
c(t)=\frac{1-e^{i \varphi t}}{n_{1} e^{i \varphi t}-n_{2}} .
$$

(ii) The pure Dresselhaus coupling $(\alpha=0, \beta \neq 0)$ :

In this case, we have

$$
\text { if } /|f|=i e^{i \omega t}, \quad h_{0}=\frac{1}{2 \beta R}
$$

and

$$
\phi(t)=\varphi t, \quad \varphi=\omega \sqrt{1+4 \beta^{2} R^{2}} .
$$

Hence we get

$$
a(t)=i e^{i \omega t} \frac{e^{i \varphi t}-1}{n_{1} e^{\varphi t}-n_{2}},
$$

where

$$
n_{1}, n_{2}=-\frac{1}{2 \beta R} \pm \frac{1}{2 \beta R} \sqrt{1+4 \beta^{2} R^{2}} .
$$

Use of

$$
H_{-}=i \beta R \hbar \omega e^{-i \omega t},
$$

and (59) leads to

$$
e^{b(t)}=\frac{\left(n_{1}-n_{2}\right)^{2} e^{i(\varphi+\omega) t}}{\left(n_{1} e^{i \varphi t}-n_{2}\right)^{2}},
$$

and

$$
c(t)=-i \frac{1-e^{i \varphi t}}{n_{1} e^{i \varphi t}-n_{2}} .
$$

\section{CONCLUSION}

In the present paper we have considered spin manipulation via the non-Abelian Berry phase induced by an adiabatic transport of a single spin along a circular path in the 2D plane in the presence of the Rashba and Dresselhaus spin-orbit couplings. We have adopted the Feynman disentangling technique to calculate the spin-flip probability. We have shown that the problem can be solved exactly in three cases: (i) the pure Rashba coupling, (ii) the pure Dresselhaus coupling, and (iii) the symmetric combination of Rashba and Dresselhaus couplings. For an arbitrary combination of the two couplings, we have carried out numerical simulations. We have plotted the spin-flip probability versus the angle of the adiabatic rotation with various values of the electric field and the radius of the circular path in the $2 \mathrm{D}$ plane. We have observed that a complete spin-flip (a complete spin precession) occurs only when the strength of the two couplings becomes equal. The relation between the complete spin precession and the persistent spin helix will be discussed in detail elsewhere. We have also obtained analytical expressions of the non-Abelian Berry phase for the pure Rashba case and the pure Dresselhaus case.

\section{Appendix A: The spin transition probabilities}

Following Popov's procedure,$\stackrel{9.18}{,}$ we show that the spin-flip probability can be expressed in the form of (41). Since the time-evolution of the spin state can be achieved by a time-dependent rotation, the transition amplitude for spin $\sigma$ to $\sigma^{\prime}$ is given by

$$
\left\langle\sigma|U(t)| \sigma^{\prime}\right\rangle=D_{\sigma, \sigma^{\prime}}^{s}(\varphi, \vartheta, \phi)=\exp \left[-i\left(\sigma \varphi+\sigma^{\prime} \phi\right)\right] d_{\sigma \sigma^{\prime}}(\vartheta) .
$$

Here $\varphi, \vartheta, \phi$ are the time-dependent Eulerian angles, $D_{\sigma, \sigma^{\prime}}^{s}(\varphi, \vartheta, \phi)$ are the elements of the Wigner $D$-matrix being the irreducible unitary representations of $S U(2)$ group, and $d_{\sigma \sigma^{\prime}}(\vartheta)$ is Wigner's $d$-function.

The corresponding transition probability along the $z$ axis is

$$
w_{\sigma \sigma^{\prime}}=\left|d_{\sigma \sigma^{\prime}}^{s}(\vartheta)(t)\right|^{2} .
$$


In particular, the transition probability from spin $1 / 2$ to $\pm 1 / 2$ is

$$
w_{1 / 2,1 / 2}=\cos ^{2}\left(\frac{\vartheta(t)}{2}\right)
$$

and

$$
w_{1 / 2,-1 / 2}=\sin ^{2}\left(\frac{\vartheta(t)}{2}\right)
$$

because

$$
d_{1 / 2,1 / 2}^{1 / 2}(\vartheta)=\cos \frac{\vartheta}{2}, \quad d_{1 / 2,-1 / 2}^{1 / 2}(\vartheta)=i \sin \frac{\vartheta}{2} .
$$

For spin $s=1 / 2$, the rotation matrix is given in the standard form, 18,19

$$
\mathbf{D}(\varphi, \vartheta, \psi)=\left(\begin{array}{cc}
\tilde{\alpha} & -\tilde{\beta}^{*} \\
\tilde{\beta} & \tilde{\alpha}^{*}
\end{array}\right),
$$

where

$$
\tilde{\alpha}=\cos \frac{\vartheta}{2} \exp \left[i \frac{\psi+\varphi}{2}\right], \tilde{\beta}=i \sin \frac{\vartheta}{2} \exp \left[i \frac{\psi-\varphi}{2}\right] .
$$

Comparison of the evolution operator for the spin $1 / 2$ transition expressed in the matrix form,

$$
\mathbf{U}=\left(\begin{array}{cc}
e^{b / 2}+a c e^{-b / 2} & a e^{-b / 2} \\
c e^{-b / 2} & e^{-b / 2}
\end{array}\right)
$$

and the rotation matrix yields

$$
|a|^{2}=\tan ^{2} \frac{\vartheta}{2} .
$$

Again comparing this result with (A3) and (A4), we arrive at

$$
w_{1 / 2,1 / 2}=\frac{1}{1+|a|^{2}}, \quad w_{1 / 2,-1 / 2}=\frac{|a|^{2}}{1+|a|^{2}} .
$$

Note that $w_{1 / 2,1 / 2}+w_{1 / 2,-1 / 2}=1$.

\section{Appendix B: Special solutions of the $\alpha-\beta$ Riccati equation}

Here we wish to solve under a special condition the Riccati equation (38):

$$
\frac{d a(t)}{d t}=-R \omega\left\{f(t)+f^{*}(t) a^{2}(t)\right\}
$$

where

$$
f(t)=\beta e^{i \omega t}+i \alpha e^{-i \omega t}, \quad f^{*}(t)=\beta e^{-i \omega t}-i \alpha e^{i \omega t} .
$$

This equation contains the Rashba limit $(\alpha \neq 0, \beta=0)$, and the Dresselhaus limit $(\alpha=0, \beta \neq 0)$, both of which have exact solutions.

First we let $a(t)=g(t) X(t)$ in (B1). If we further let $g(t)=-i f /|f|$, then we see that $X(t)$ obeys

$$
\frac{d X}{d t}=i R \omega|f(t)|\left\{X^{2}-2 h(t) X-1\right\},
$$

where

$$
|f(t)|=\left[\alpha^{2}+\beta^{2}+2 \alpha \beta \sin (2 \omega t)\right]^{1 / 2}
$$

and

$$
h(t)=\frac{\beta^{2}-\alpha^{2}}{2 R\left(\alpha^{2}+\beta^{2}\right)^{3 / 2}}\left[1+\frac{2 \alpha \beta}{\alpha^{2}+\beta^{2}} \sin (2 \omega t)\right]^{-3 / 2} .
$$

Now we consider a special case where $h(t)$ is a constant, say, $h_{0}$. In this case, (B3) can be expressed as

$$
\frac{d X}{\left(X-n_{1}\right)\left(X-n_{2}\right)}=i R \omega|f(t)| d t,
$$

where $n_{1}$ and $n_{2}$ are roots of

$$
X^{2}-2 h_{0} X-1=0,
$$

that is,

$$
n_{1}, n_{2}=h_{0} \pm \sqrt{h_{0}^{2}+1} .
$$

Note that

$$
n_{1} n_{2}=-1, \quad n_{1}+n_{2}=2 h_{0}, \quad n_{1}-n_{2}=2 \sqrt{h_{0}^{2}+1} .
$$

Upon integration, we obtain with the condition $X(0)=0$,

$$
X(t)=-\frac{1-e^{i \phi(t)}}{n_{2}-n_{1} e^{i \phi(t)}}
$$

The phase function $\phi(t)$ is

$$
\phi(t)=R \omega\left(n_{1}-n_{2}\right) \int_{0}^{t}|f(\tau)| d \tau
$$

which can be expressed in closed form,

$$
\begin{aligned}
\phi(t)= & 2 R \omega \sqrt{h_{0}^{2}+1}(\alpha+\beta) \\
& \left\{E\left(\omega t-\frac{\pi}{4}, \frac{2 \sqrt{\alpha \beta}}{\alpha+\beta}\right)-E\left(-\frac{\pi}{4}, \frac{2 \sqrt{\alpha \beta}}{\alpha+\beta}\right)\right\},(
\end{aligned}
$$

where $E(\varphi, k)$ is the elliptic function of the second kind defined by

$$
E(\varphi, k)=\int_{0}^{\varphi} \sqrt{1-k^{2} \sin ^{2} \theta} d \theta
$$


Consequently, for the case where $h(t)=h_{0}$, the starting Riccati equation (B1) is exactly solved, the result being of the form,

$$
a(t)=\frac{i f}{|f|} \frac{e^{i \phi}-1}{n_{1} e^{i \phi}-n_{2}} .
$$

Since $\phi(0)=0$, it is evident that $a(0)=0$. Using (B8) in (B13), we obtain

$$
|a(t)|^{2}=\frac{\sin ^{2}(\phi / 2)}{h_{0}^{2}+1-\sin ^{2}(\phi / 2)} .
$$

The transition probabilities from spin $1 / 2$ to $\pm 1 / 2$ are given by

$$
w_{1 / 2,1 / 2}=1-\frac{1}{h_{0}^{2}+1} \sin ^{2}\left(\frac{\phi(t)}{2}\right)
$$

and

$$
w_{1 / 2,-1 / 2}=\frac{1}{h_{0}^{2}+1} \sin ^{2}\left(\frac{\phi(t)}{2}\right)
$$

which are characterized only by the constant $h_{0}$ and the phase function $\phi(t)$.

Although the above results are exact under the assumption that $h(t)=h_{0}$ is a constant, they are approximate results when $h(t) \approx h_{0}$.

Finally, specifying the values of $h_{0}$ and $\phi(t)$, we shall obtain the exact results for the Rashba, the Dresselhaus and the symmetric cases.

(i) The Rashba limit $(\alpha \neq 0, \beta=0)$ : In this case, from (B5) follows

$$
h_{0}=-\frac{1}{2 \alpha R \omega} \text {. }
$$

Furthermore the right-hand side of (B11) can be easily integrated, so that

$$
\phi(t)=\sqrt{1+4 \alpha^{2} R^{2}} \omega t .
$$

Thus the spin flip probability is obtained in the form,

$$
w_{1 / 2,-1 / 2}^{R}=\frac{4 \alpha^{2} R^{2}}{1+4 \alpha^{2} R^{2}} \sin ^{2}\left\{\frac{1}{2} \sqrt{1+4 \alpha^{2} R^{2}} \theta\right\},
$$

where $\theta=\omega t$.

(ii) The Dresselhaus limit $(\alpha=0, \beta \neq 0)$ : In this case, (B5) leads to

$$
h_{0}=\frac{1}{2 \beta R \omega} \text {. }
$$

The integral of (B11) yields

$$
\phi(t)=\sqrt{1+4 \beta^{2} R^{2}} \omega t .
$$

Hence the spin-flip probability is

$$
w_{1 / 2,-1 / 2}^{D}=\frac{4 \beta^{2} R^{2}}{1+4 \beta^{2} R^{2}} \sin ^{2}\left\{\frac{1}{2} \sqrt{1+4 \beta^{2} R^{2}} \theta\right\}
$$

(iii) The symmetric case $(\alpha=\beta \neq 0)$ : In this particular case,

$$
h_{0}=0 \text {. }
$$

The phase factor becomes

$$
\phi(t)=2 \sqrt{2} \alpha R[\sin (\omega t)-\cos (\omega t)+1] .
$$

The corresponding spin-flip probability as a function of $\theta=\omega t$ is

$$
w_{1 / 2,-1 / 2}^{\text {sym }}=\sin ^{2}\{\sqrt{2} \alpha R[\sin \theta-\cos \theta+1]\} .
$$

\section{ACKNOWLEDGMENTS}

This work was supported by the NRI INDEX center, USA, NSERC and CRC program, Canada.
1 M. V. Berry, Proc. Roy. Soc. London, Series A, Math. Phys. Sci., 392, 45 (1984).

2 F. Wilczek and A. Shapere Geometric Phases in Physics, Wold Scientific (1989).

3 D. Loss and D. P. DiVincenzo, Phys. Rev. A 57, 120(1998); G. Burkard, D. Loss and D. P. DiVincenzo, Phys. Rev. B 59, 2070 (1999).
4 J. A. Jones, V. Vedral, A. Ekert and G. Castagnoli, Nature 403, 869 (2000);G. Falci, R. Fazio, G. M. Palma, J. Siewert ad V. Vedral, Nature 407, 355(2000).

5 X. Hu and S. Das Sarma, Phys. Rev. A 61, 062301 (2000).

${ }^{6}$ P. San-Jose, G. Zarand, A. Shnirman and G. Schön, Phys. Rev. Lett. 97, 076803 (2006); P. San-Jose, G. Schön, A. Shnirman and G. Zarand, Physica E: Low-dimensional Sys- 
tems and Nanostructures 40, 76 (2007); P. San-Jose, B. Scharfenberger, G. Schön, A. Shnirman, G. Zarand, Phys. Rev. B 77, 045305 (2008).

7 H. Wang, K.-D. Zhu, EPL 82, 60006 (2008).

8 R. P. Feynman, Phys. Rev. 84, 108(1951).

9 V. S. Popov, J. Exptl. Theoret. Phys. (U.S.S.R.) 35, 985 (1958);[Sov. Phys. JETP 8, 687 (1959)].

10 Y. A. Bychkov and E. I. Rashba, J. Phys. C 17, 6039 (1984).

11 G. Dresselhaus, Phys. Rev. 100, 580 (1955).

12 W. T. Reid, Riccati Differential Equations (Academic Press, New York, 1972).

13 S. Prabhakar, J. E. Raynolds, A. Inomata, SPIE 7720, 77020V (2010).

14 R. de Sousa, S. Das Sarma, Phys. Rev. B 68, 155330 (2003).

15 S. Prabhakar and J. E. Raynolds, Phys. Rev. B 79, 195307 (2009).

16 F. Wilczek and A. Zee, Phys. Rev. Lett. 52, 2111 (1984).
17 M. Pletyukhov and O. Zaitsev, Journal of Physics A: Mathematical and General 36, 5181 (2003);O. Zaitsev, D. Frustaglia and K. Richter, Phys. Rev. B 72, 155325 (2005).

18 V. S. Popov, Phys.-Usp. 50, 1217 (2007).

19 A. Inomata, H. Kuratsuji and C. C. Gerry, Path Integrals and Coherent States of SU(2) and SU(1,1), World Scientific, Singapore (1992).

20 I. L. Aleiner and V. I. Fal'ko, Phys. Rev. Lett. 87, 256801 (2001).

21 S.-H. Chen and C.-R. Chang, Phys. Rev. B 77, 045324 (2008).

22 B. A. Bernevig, J. Orenstein and S.-C. Zhang, Phys. Rev. Lett. 97, 236601 (2006).

23 M.-H. Liu, C.-R. Chang and S.-H. Chen, Phys. Rev. B 71, 153305 (2005); M.-H. Liu, K.-W. Chen, S.-H. Chen and C.-R. Chang, Phys. Rev. B 74, 235322 (2006).

24 J. D. Koralek, C. P. Weber, J. Orenstein, B. A. Bernevig, S.-C. Zhang, S. Mack and D. D. Awschalom, Nature 458, 610 (2009). 